Jianjun Lin, Huisu Chen*, Zhong Lv and Yujiang Wang

\title{
Analytical solution on dosage of self-healing capsules in materials with two-dimensional multi-shaped crack patterns
}

https://doi.org/10.1515/secm-2017-0256

Received July 28, 2017; accepted February 23, 2018; previously published online April 23, 2018

\begin{abstract}
This article presents a numerical method for determining the dosage of pre-embedded capsules in selfhealing materials with complex crack patterns. The crack distribution on the surface of materials is simplified into a two-dimensional (2D) multi-shaped geometrical structure composed of triangles, rhombuses, and hexagons with specified area fractions, and further decomposed into three separate mono-shaped crack systems. Then, the dosage of capsules required to heal the cracks in each monoshaped crack system is computed. According to the area fraction of each mono-shaped polygon in the whole system, the integrated models of crack-hitting probability by the capsules and the capsule dosage for the multi-shaped crack system are derived. The analytical results reveal that the dosage of capsules significantly depends on the spatial distribution of the cracks and the ratio of the capsule length to the crack size. For a certain fixed crack pattern, the size and dosage of capsules will strongly affect crack healing efficiency.
\end{abstract}

Keywords: capsule dosage; geometrical probability; linear crack patterns; self-healing materials.

\section{Introduction}

Cracks are one of the critical factors that induce loss in the load-bearing capacity and durability of structures [1-3]. To reduce the negative impact of cracks on structural properties, inspired by the autogenous healing of

\footnotetext{
*Corresponding author: Huisu Chen, Jiangsu Key Laboratory of Construction Materials, School of Materials Science and Engineering, Southeast University, Nanjing 211189, P.R. China, Phone: +86-13270890362, e-mail: chenhs@seu.edu.cn Jianjun Lin and Yujiang Wang: Jiangsu Key Laboratory of Construction Materials, School of Materials Science and Engineering, Southeast University, Nanjing 211189, P.R. China Zhong Lv: School of Architectural Engineering, Anhui University of Technology, Ma'anshan 243032, P.R. China
}

organs in creatures, a self-healing concept was proposed, and the related techniques [4-6] were then developed in many fields such as biomaterials [7-9] and organic/ inorganic materials $[10,11]$. Depending on the method used to deliver the healing components to the target site, self-healing techniques can be roughly divided into two categories: intrinsic and extrinsic. The former operates through inter/intra-macromolecular interaction, and the latter occurs with the aid of an embedded healing agent. With technological advancements, self-healing based on microencapsulated healing agents has become the most common approach for crack healing in recent years and is more likely to be commercialized because of its practicability and simplicity [12-15].

In actual projects, the amount of healing agent is an important factor affecting the reliability and service life of materials and often quantified by both the number of capsules per unit volume and the capsule size $[16,17]$. To know the relationship between the volume fraction of capsules and healing efficiency, many experiments and theoretical approaches on the volume fraction of capsules required in self-healing materials have been considered. For example, Huang et al. [18] investigated self-healing in cementitious materials with saturated $\mathrm{Ca}(\mathrm{OH})_{2}$ solution as the healing agent supplied by capsules and a vascular system, and the influence of capsule dosage and size was taken into account. In Ref. [19], self-healing due to further hydration of unhydrated cement particles was taken as an example for investigating the effects of capsules on the self-healing efficiency and mechanical properties of cementitious materials. The efficiency of supply of water by using capsules as a function of capsule dosage and size was determined numerically. Yuan and Chen [20] applied geometrical probability to derive a statistical model for determining the length of capsule for a given simple crack pattern, as well as a model for calculating the proper dosage of capsules. Lv et al. [21-23] proposed a series of geometrical patterns such as parallel linear, rectangular, parallel planar, and so on, and developed the corresponding models of capsule dosage on the basis of the above crack patterns. Previous studies [20-23] have shown that the required number of capsules in self-healing materials greatly depends on the distribution of cracks. 

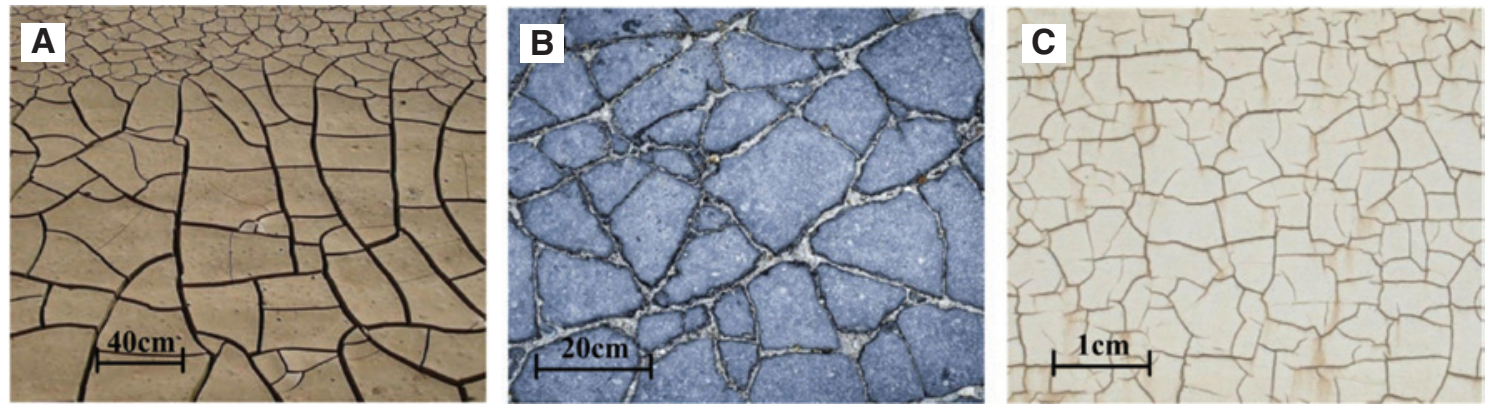

Figure 1: Actual cracks on the surface of structures: (A) cracked soil, (B) cracked asphalt pavement, and (C) cracked wall coating.

From the literature on sulfate attack [24], drying shrinkage [25], alkali aggregate reaction [26], and freezethaw cycles [27] of cement-based materials, it is not hard to find that the crack configuration in those materials is very complicated and diverse due to various degradation mechanisms. For cracking structures such as cracked soil, organic/inorganic coatings [28, 29], and concrete pavements [30,31], the generated cracks exhibit as a complex geometrical configuration, which is analogous to a network composed of multi-shaped cells (Figure 1). Thus, it is necessary to develop a methodology for determining the required dosage of pre-embedded capsules in materials with more complex geometric crack patterns than before.

In this study, the concept and deducing method of the interaction probability between capsules and cracks are presented first. Taking the linear crack distribution in Figure 1 as the research foundation, a simplified 2D multi-shaped geometrical structure composed of triangles, rhombuses, and hexagons is introduced. The multishaped crack pattern is decomposed into three separate mono-shaped crack systems. By combining the formulas in Section 2, the required dosage of capsules in each mono-shaped system is computed. Afterward, according to the area fraction of each polygon in the whole system, the crack-hitting probabilities by the capsules for the multi-shaped crack pattern are computed and the models of capsule dosage are derived. Computer simulation is also employed to verify the reliability of these models.

\section{Materials and methods}

\subsection{The binomial distribution}

In general, to reduce the potentially negative effect of the addition of capsules on the overall macro properties of materials, the volume of capsules embedded in materials should not be too high, and it should preferably be less than $5 \%$ of the matrix [32]. Under these conditions, the capsules form a stationary, random system of nonoverlapping geometric objects. We assume that all the healing capsules are randomly dispersed in the material and the cracks appear in a certain pattern. The process of tossing capsules onto the crack network will produce two results: one is the capsule intersecting with cracks, and the other resulting in no intersection. This reminds us of the Buffon needle problem - a classical problem of the probability of a randomly tossed needle intersecting with a set of parallel lines in a plane [33].

Taking the needles' analogy for the capsules, the geometrical relationship between the needles and cracks is complicated and their independence may be hard to find in practice. But if the scale of the matrix is large enough, the independence between needles and cracks can be assumed, and this assumption can be used in our models to simplify the problem. The length of the healing capsules in materials is normally fixed, while the size of cracks always propagates and some extra cracks may form with the increase of service time and external load. That is, at different stages of service life of the structures, the ratio of the capsule length to the crack size may vary, which may influence the crackhitting probabilities by the capsules. Therefore, it is necessary to consider all possible conditions of different ratios of the capsule length to the crack size.

Based on the assumption of independence, intersection or not between capsules and cracks is regarded as a Bernoulli probability variable, and the number of successes is represented by a binominal distribution. In what follows, we will briefly present the concept of binomial distribution [34].

From the statistical point of view, the binomial distribution is the discrete probability distribution of the number of successes in a sequence of $m$ independent yes/ no trials. For a random variable $X$, which is defined as the number of successes in $m$ trials, if the following conditions are met, the variable $X$ has a binomial distribution with parameters $m$ and $p$, abbreviated as $B(m, p)$ : 
(a) The experiment consists of $m$ identical trials and all the trials are independent.

(b) Each trial results in one of two outcomes. One of the outcomes is called a success (S), and the other a failure $(\mathrm{F})$.

(c) The probability of success or failure on a single trial is equal to $p$ and $1-p$, respectively.

For capsules containing the healing agent and cracks in materials, if there are $N$ capsules and the crack-hitting probabilities by the capsules $p$ stay the same, the random variable $X$ denoting the total number of intersection in the area follows the binomial distribution, and the mean of $X$, denoted by $E(X)$, can be expressed by Eq. (1).

$$
E(X)=N \cdot p
$$

Assume that each capsule has the capability for repairing cracks of length equal to the threshold $L_{\text {heal }}$. The expected length of repairing the crack by $N$ capsules can be expressed as $L_{\text {heal }} E(X)$. The total length of cracks in the material is $L_{T}$. If those cracks can be completely repaired, the following relationship (i.e. Eq. 2) should be fulfilled:

$$
L_{\text {heal }} E(X) \geq L_{T}
$$

\subsection{Hitting probabilities}

In order to better deal with the problems of hitting probability and expand the range of its application, the definition of kinetic measure is applied to the geometric probabilities. In the literature [35], the following theorem is made use of to derive the kinetic measure of a segment contained in an arbitrary convex cell:

Theorem 1: Let $K$ be a bounded convex cell of perimeter $L$ and area $A$, and there is a segment of length $l$ in the plane. The kinematic measure of the segment contained in the domain $K$ is

$$
m(l)=\pi A-\int_{0}^{2 \pi} d \varphi \int_{0}^{r(l, \varphi)} p(\sigma, \varphi) d \sigma
$$

where $m(l)$ is the kinematic measure of a segment with length $l ; p(\sigma, \varphi)$ and $r(l, \varphi)$ are the generalized support function and the restricted chord function of the convex cell $K$, respectively; and $\varphi$ is an angle between 0 and $2 \pi$. For the definition and derivation of $p(\sigma, \varphi)$ and $r(l, \varphi)$, refer to [35].

For an arbitrary convex cell $K$ [35], the hitting probability of the line segment with the boundary of cell $K$ can be derived by substituting Eq. (3) into Eq. (4):

$$
p=1-\frac{m(l)}{\pi A}
$$

\section{Simplified crack pattern}

Roughly, the distribution of linear cracks in Figure 1 can be simplified to an edge system of a variety of tessellations. The models of Voronoi tessellations [36], Laguerre tessellations [37], and the random tessellations that are stable under the operation of iteration (STIT tessellations) $[38,39]$, which can generate various irregular convex polygons, are commonly used as geometric models for crack patterns. However, because of their complexity, it is difficult to apply those models. To show how the analytical solution of capsule dosage is derived for a relatively complex crack system, here we consider only some regular polygons as examples. As shown in Figure 2, a simplified 2D multi-shaped crack pattern composed of triangles, rhombuses, and hexagons with specified area fractions and arrangement form is employed: the areas of the triangle, rhombus, and hexagon in the hypothetical crack system are $\sqrt{3} a^{2} / 4, \sqrt{3} a^{2} / 2$, and $3 \sqrt{3} a^{2} / 2$, respectively, where $a$ is the side length of those polygons. All the randomly distributed capsules in the plane are idealized as $1 \mathrm{D}$ linear segments with a fixed length $l$ and negligible thickness. Each linear capsule is capable of repairing cracks of length equal to the threshold $L_{\text {heal }}$.

To obtain the analytical solutions of the crack-hitting probability by the capsules and the capsule dosage in the complex crack system, we need to obtain the analytical models in each mono-shaped crack system first. The multi-shaped crack configuration is decomposed into three separate mono-shaped crack systems (i.e. triangular, rhombic and hexagonal crack patterns). It is noteworthy that small differences in the crack distribution may have a considerable impact on determining the dosage of selfhealing capsules in materials. Accordingly, the outcomes

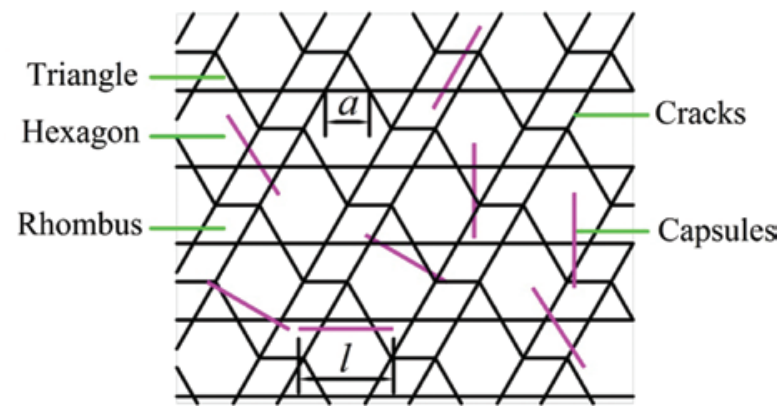

Figure 2: A triple-shaped crack distribution composed of triangles, rhombuses, and hexagons. 
and conclusions presented herein are valid only within the present selections of crack patterns and capsules.

\subsection{The mono-shaped crack patterns}

\subsubsection{Triangular crack pattern}

Assume that there exist three sets of equidistant, parallel, linear cracks on the surface of materials, and the parallel cracks form the regular triangular crack pattern as schematically shown in Figure 3. The kinematic measure of a linear capsule in a regular triangular cell is described in the Appendix [40]. According to Eq. (4) and the ratio of

$$
L_{T}=\frac{3 a}{2} n(n+1)
$$

If those cracks can be repaired, the required number of capsules $N$ should fulfill Eq. (2). Substituting Eqs. (5-7) into Eq. (2) yields Eq. (8):

$$
N_{A} \geq \frac{6}{\sqrt{3} a L_{\text {heal }}} \cdot \frac{1}{p_{T}}
$$

where $N_{A}$ is the required number of capsules per unit area of the materials, and equivalent to $N / A_{T}$ [41]. Therefore, for the triangular crack pattern in Figure 3, Eq. (8) should be fulfilled if all of the cracks are to be completely repaired.

\subsubsection{Rhombic crack pattern}

$p_{R}= \begin{cases}\frac{72 a l-18 l^{2}-\sqrt{3} \pi l^{2}}{9 \sqrt{3} \pi a^{2}}, & \frac{l}{a} \leq \frac{\sqrt{3}}{2} \\ \frac{72 a l-18 l^{2}-\sqrt{3} \pi l^{2}-45 a \sqrt{4 l^{2}-3 a^{2}}+12 \sqrt{3}\left(l^{2}+3 a^{2}\right) \arccos \frac{\sqrt{3} a}{2 l}}{9 \sqrt{3} \pi a^{2}}, & \frac{\sqrt{3}}{2}<\frac{l}{a} \leq 1 \\ \frac{(2 \sqrt{3} \pi+9)\left(3 a^{2}+2 l^{2}\right)-27 a \sqrt{4 l^{2}-3 a^{2}}+12 \sqrt{3}\left(3 a^{2}-l^{2}\right) \arccos \frac{\sqrt{3} a}{2 l}}{18 \sqrt{3} \pi a^{2}}, & 1<\frac{l}{a} \leq \sqrt{3} \\ 1.0, & \sqrt{3}<\frac{l}{a}\end{cases}$

the capsule length to the crack size $(l / a)$, the crack-hitting probability $p_{\mathrm{T}}$ by the capsules can be expressed as Eq. (5):

$p_{T}=\left\{\begin{array}{lc}\frac{12 \sqrt{3} a l-2 \pi l^{2}-3 \sqrt{3} l^{2}}{3 \pi a^{2}}, & \frac{l}{a} \leq \frac{\sqrt{3}}{2} \\ \frac{12 l-9 \sqrt{4 l^{2}-3 a^{2}}}{\sqrt{3} \pi a}-\frac{2 l^{2}}{3 a^{2}}-\frac{\sqrt{3} l^{2}}{\pi a^{2}}+\frac{4 l^{2}+6 a^{2}}{\pi a^{2}} \arccos \frac{\sqrt{3} a}{2 l} & , \frac{\sqrt{3}}{2}<\frac{l}{a} \leq 1 \\ 1.0, & 1<\frac{l}{a}\end{array}\right.$

The event that a capsule intersects with the cracks is a binomial experiment, and the number of capsules intersecting with the cracks is the binomial random variable $X$. A representative region $E 1$ with side length $n a$ (Figure 3) is selected with the edges coinciding with the cracks, and has an area $A_{T}$ :

$$
A_{T}=n^{2} \frac{\sqrt{3}}{4} a^{2}
$$

where $n^{2}$ is the number of regular triangles in the region $E 1$, and $A_{T}$ is the area of the region $E 1$. The total length $L_{T}$ of cracks can be represented by Eq. (7):
A rhombic crack pattern on the surface of materials is shown in Figure 4. The angles between the crossed linear cracks are $60^{\circ}$. The kinematic measure of linear capsule in the rhombic cell is described in the Appendix [40]. According to the ratio of the capsule length to the crack size $(l / a)$, the crack-hitting probability $p_{R}$ by the capsules [i.e. Eq. (9)] can be obtained by Eq. (4).

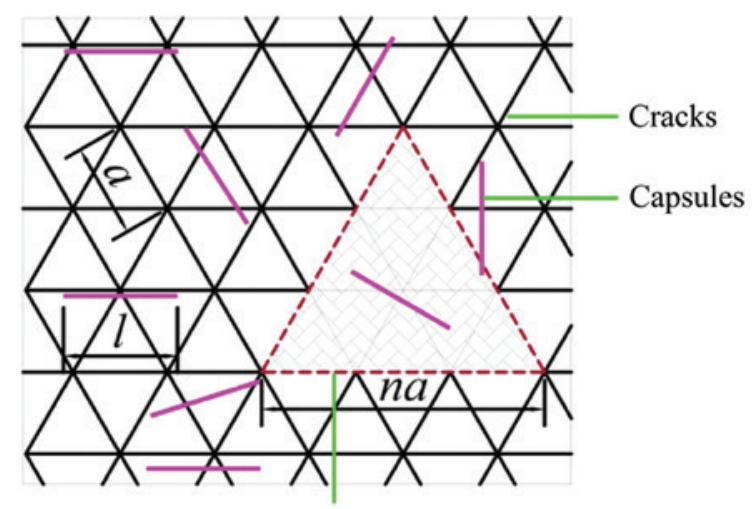

Figure 3: Triangular crack pattern and a representative sampling region $E 1$. 


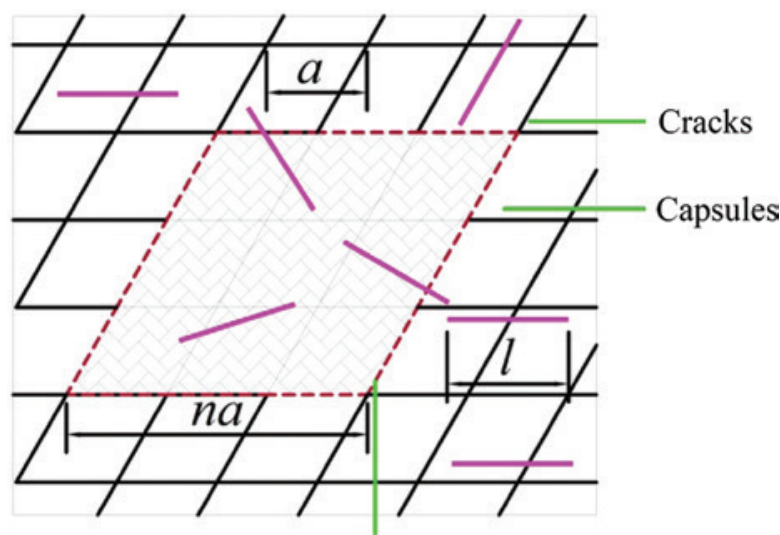

Figure 4: Rhombic crack pattern and a representative sampling region $E 2$.

$$
\begin{gathered}
A_{T}=n^{2} \frac{\sqrt{3}}{2} a^{2} \\
L_{T}=2 \operatorname{an}(n+1)
\end{gathered}
$$

A rhombic region E2, which is composed of $n^{2}$ rhombuses, is selected as a representative sampling region, with the edges coinciding with the linear cracks. The area of the region $A_{T}$ and length of cracks inside $L_{T}$ are obtained by Eqs. (10) and (11), respectively. The required number of capsules per unit area of materials for rhombic crack pattern [i.e. Eq. (12)] is obtained by substituting Eqs. (9-11) into Eq. (2).

$$
N_{A} \geq \frac{4}{\sqrt{3} a L_{\text {heal }}} \cdot \frac{1}{p_{R}}
$$

\subsubsection{Hexagonal crack pattern}

Another mono-shaped crack system - hexagonal crack pattern - is formed here by a large number of line segments. The side length of a regular hexagon and length of the capsules are given in Figure 5. The kinematic measure of linear capsule in the hexagonal cell is also described in the Appendix [40]. The crack-hitting probability by the capsules $p_{H}$ is expressed as Eq. (13):

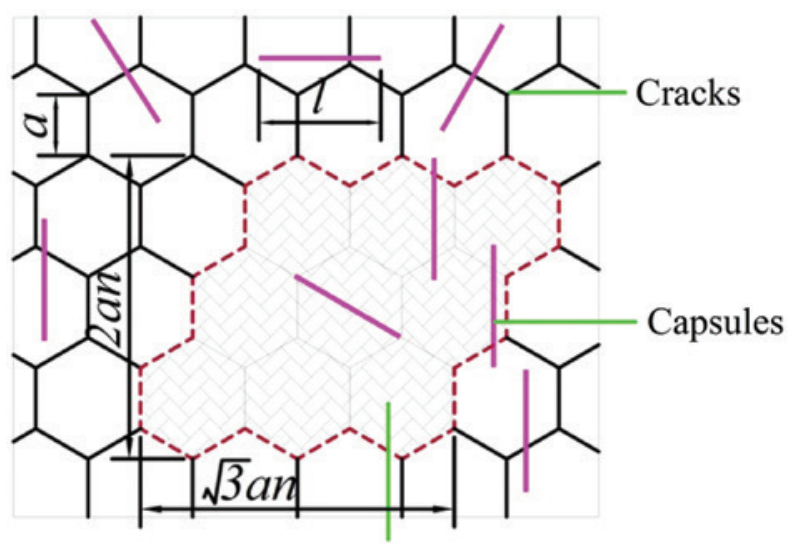

Figure 5: Hexagonal crack pattern and a representative sampling region E3.

In Figure 5, a complex sampling region E3 is selected, and $n^{2}$ regular hexagons exist in the region. The area of region $A_{T}$, and length of cracks $L_{T}$, are obtained by Eqs. (14) and (15), respectively.

$$
\begin{gathered}
A_{T}=n^{2} \frac{3 \sqrt{3}}{2} a^{2} \\
L_{T}=\left(3 n^{2}+4 n-1\right) a
\end{gathered}
$$

Referring to the derivation of $N_{A}$ in Section 3.1.1, the solution of capsule dosage is derived, and it is expressed as Eq. (16):

$$
N_{A} \geq \frac{2}{\sqrt{3} a L_{\text {heal }}} \cdot \frac{1}{p_{H}}
$$

\subsubsection{Verification of crack-hitting probability models}

The verification algorithm is done as follows:

(a) Generate three kinds of polygonal cells with the side length $a$ (Figure 6).

(b) Randomly generate a number of capsules $N_{i}(i=1$, $2, \ldots, m$, where $m$ is set by user) with length inside above polygonal cells, the location of capsules is determined by its mid-point coordinate $\left(x_{i}, y_{i}\right)$ as well

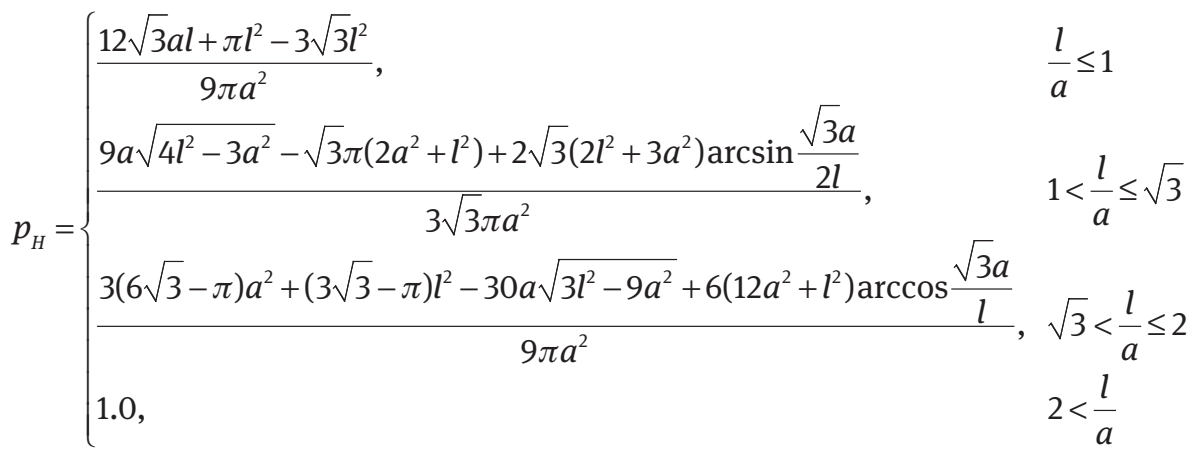



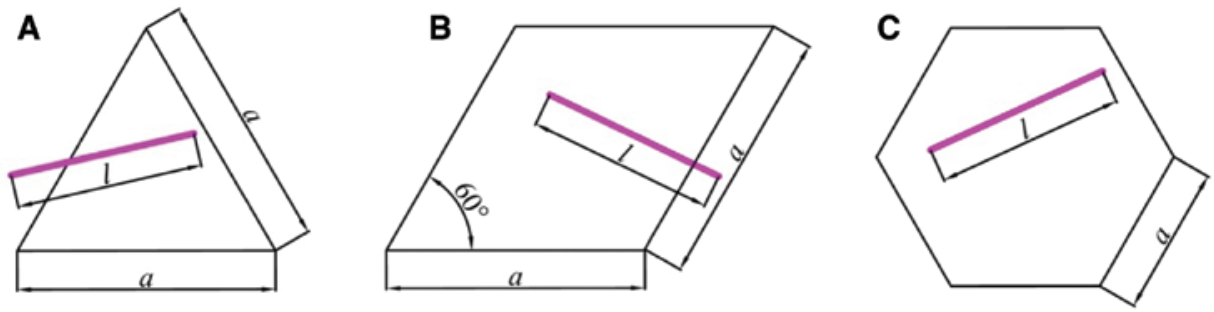

Figure 6: Polygonal cells of different crack patterns: (A) triangle, (B) rhombus, and (C) hexagon.

as the orientation angle $\theta_{i}$. The value $l / a$ is separately selected to be equal to $0.50,0.87,1.10$ or 1.80 , which is located in the specified ranges as listed in Table 1.

(c) Count the number of capsules intersecting with the boundary of cells $k$ and the total number of capsules $m$. The crack-hitting probability $p$ by the capsules can be estimated as the ratio

$$
p=\frac{k}{m}
$$

Simulations from triangular, rhombic, and hexagonal crack patterns at different ratios of the capsule length to the crack size are compared with the theoretical values. It can be seen that (Figure 7) with the increase of the tossed number $m$ of the capsules $m$, the simulated probability $p$ tends to a stable value and is consistent with the theoretical value, which verifies the reliability of the models for the crack-hitting probability by the capsules.

\subsubsection{Verification of capsule dosage models}

(a) Generate the representative sampling region $E 1, E 2$, and $E 3$ as shown in Figures 3-5, with the size $a=1.0$. The number of corresponding polygonal cells in each region is $n^{2}$, and the required number of capsules is calculated through the models of the capsule dosage $N_{A}$ [i.e. Eqs. (8), (12) and (16)].

(b) Assume that the length of cracks $L_{\text {heal }}$ that can be healed by a single capsule is equal to 0.50. Then randomly toss capsules into the region and count the number of capsules intersecting the cracks. The healing ratio of the cracks $p_{\text {heal }}$ can be obtained by Eq. (18):

$$
p_{\text {heal }}=\frac{k L_{\text {heal }}}{L_{T}}
$$

After replacing quantitative parameters of capsules and the crack pattern in the sampling region by fixed numerical values, the simulation results for capsule dosage show good consistency with theoretical solutions. As shown in Figure 8, as the number of polygonal cells $\left(n^{2}\right)$ increases, all the healing ratios $p_{\text {heal }}$ reach a stable value 1.0.

\subsection{Multi-shaped crack pattern}

All crack-hitting probability models and capsule dosage models for triangular, rhombic, and hexagonal crack patterns have been proposed and verified by numerical simulations. If the area fraction of each mono-shaped polygonal cell in the whole system is known, then the integrated model of crack-hitting probabilities by the capsules and the analytical model of capsule dosage for complex crack system can be derived.

\subsubsection{Crack-hitting probability model}

Theorem 2 [42]: Consider an arbitrary, multi-shaped system comprised of convex polygonal cells $K_{1}, K_{2}, \ldots, K_{n}$. If we randomly toss a line segment of length $l$ in the plane, the intersection probability of the line segment with the boundary of cells can be expressed as Eq. (19):

$$
p=1-\frac{m_{1}(l)+m_{2}(l)+\cdots+m_{n}(l)}{\pi\left(A_{1}+A_{2}+\cdots+A_{n}\right)}
$$

\begin{tabular}{|c|c|c|c|c|c|}
\hline Crack patterns & $\frac{l}{a} \leq \frac{\sqrt{3}}{2}$ & $\frac{\sqrt{3}}{2}<\frac{l}{a} \leq 1$ & $1<\frac{l}{a} \leq \sqrt{3}$ & $\sqrt{3}<\frac{l}{a} \leq 2$ & $2<\frac{l}{a}$ \\
\hline Triangular crack pattern & 0.50 & 0.87 & - & - & - \\
\hline Rhombic crack pattern & 0.50 & 0.87 & 1.10 & - & - \\
\hline Hexagonal crack pattern & 0.50 & - & 1.10 & 1.80 & - \\
\hline
\end{tabular}

Table 1: Selection of the ratio $l / a$ for different crack patterns. 

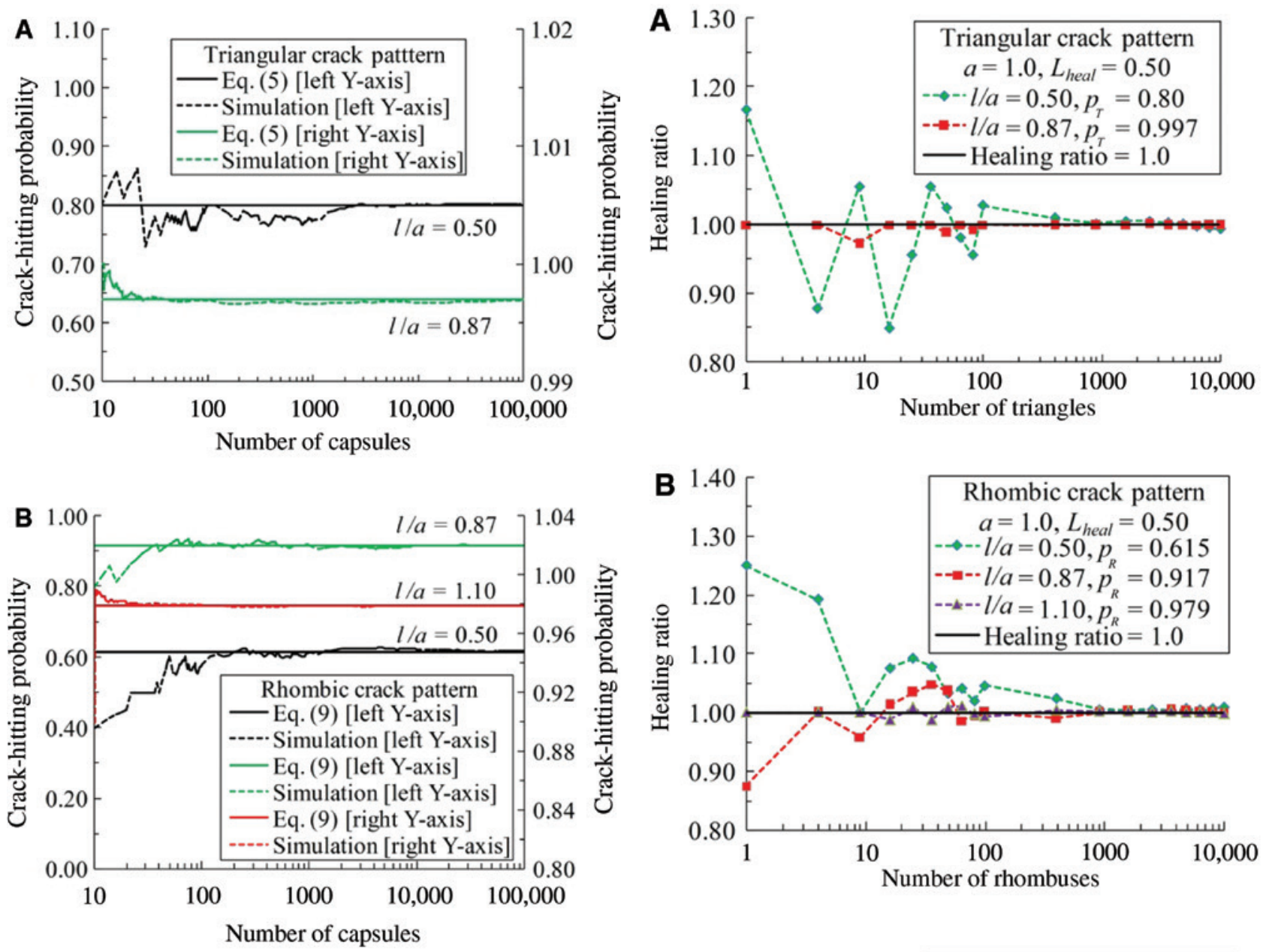

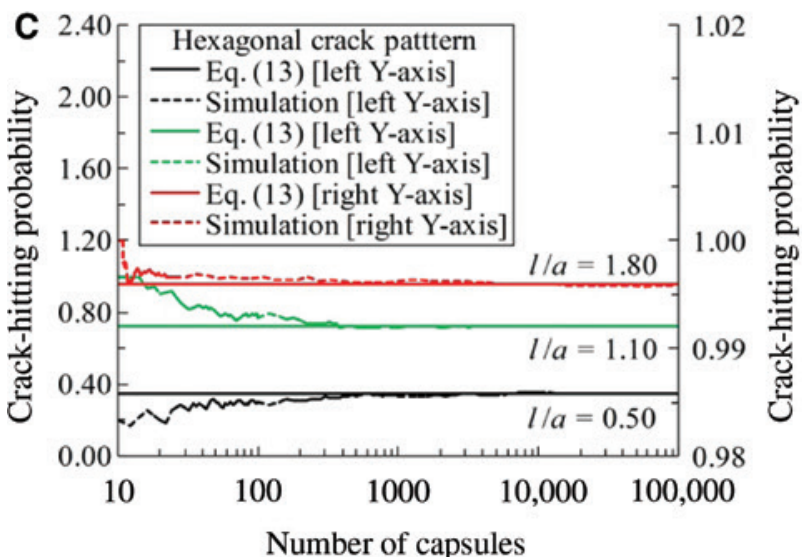

Figure 7: Verification of crack-hitting probability models for monoshaped crack patterns: (A) triangular crack pattern, (B) rhombic crack pattern, and $(C)$ hexagonal crack pattern.

where $A_{1}, A_{2}, \ldots, A_{n}$ are the area of the convex cells $K_{1}, K_{2}, \ldots, K_{n}$, and $m_{1}(l), m_{2}(l), \ldots, m_{n}(l)$ are the kinematic measure of the line segment in the corresponding cells.
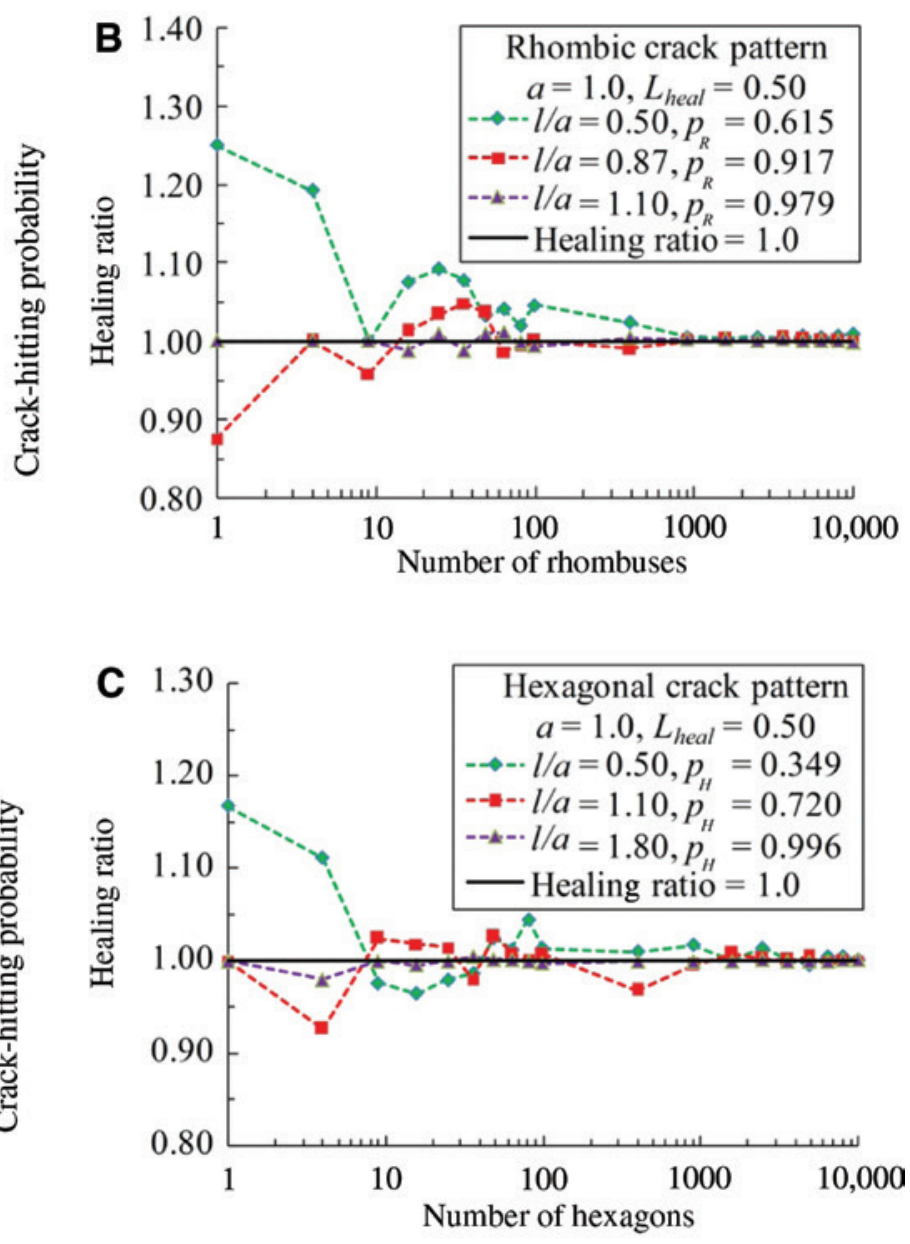

Figure 8: Verification of capsule dosage models for mono-shaped crack patterns: (A) triangular crack pattern, (B) rhombic crack pattern, and (C) hexagonal crack pattern.

By combining Eqs. (19) and (4), the intersecting probability of a line segment with the boundary of multishaped cell system can be translated into a simplified form as Eq. (20): 


$$
p=\sum_{i=1}^{n}\left(\frac{A_{i}}{\sum_{j=1}^{n} A_{j}}\right) \cdot p_{i}
$$

For the multi-shaped crack pattern in Figure 2, a combined cell composed of two regular triangles, two rhombuses, and one hexagon is selected. These polygons are arranged tightly, as shown in Figure 9, and the area of the triangular, rhombic, and hexagonal domains are, respectively, $\sqrt{3} a^{2} / 2, \sqrt{3} a^{2}$, and $3 \sqrt{3} a^{2} / 2$. Based on the area fraction of various polygons in the whole system and the ratios of the capsule length to the crack size, the crack-hitting probability $p_{c}$ by the capsules [i.e. Eq. (21)] can be derived by substituting Eqs. (8), (12), and (16) into Eq. (20): combined cells exist in total. Further, the region is also assumed to be periodic as the cell. The area of the region $E_{x y}$ is expressed by Eq. (22):

$$
A_{T}=x \cdot y=3 \sqrt{3} a^{2} n^{2}
$$

The total length of cracks $L_{T}$ is obtained by Eq. (23):

$$
L_{T}=10 a n^{2}+5 a n
$$

Substituting Eqs. (21-23) into Eq. (2) yields Eq. (24). That is to say, for the multi-shaped crack pattern, to fully heal the cracks on the surface of materials, the required number of capsules should fulfill Eq. (24):

$$
N_{A} \geq \frac{10}{3 \sqrt{3} a L_{\text {heal }}} \cdot \frac{1}{p_{c}}
$$

$$
p_{c}= \begin{cases}\frac{360 a l-5 \sqrt{3} \pi l^{2}-90 l^{2}}{54 \sqrt{3} \pi a^{2}}, & \frac{l}{a} \leq \frac{\sqrt{3}}{2} \\ \frac{90(4 a-l) l-5 \sqrt{3} \pi l^{2}-171 a \sqrt{4 l^{2}-3 a^{2}}+6 \sqrt{3}\left(10 l^{2}+21 a^{2}\right) \arccos \frac{\sqrt{3} a}{2 l}}{54 \sqrt{3} \pi a^{2}}, & \frac{\sqrt{3}}{2}<\frac{l}{a} \leq 1 \\ \frac{1}{54 \sqrt{3} \pi a^{2}}\left[3(9-\sqrt{3} \pi) a^{2}+54 a \sqrt{4 l^{2}-3 a^{2}}+12 \sqrt{3}\left(3 a^{2}-l^{2}\right) \arccos \frac{\sqrt{3} a}{2 l}\right. & 1<\frac{l}{a} \leq \sqrt{3} \\ \left.+(18-5 \sqrt{3} \pi) l^{2}+18 \sqrt{3}\left(3 a^{2}+2 l^{2}\right) \arcsin \frac{\sqrt{3} a}{2 l}\right], & \sqrt{3}, \\ \frac{6(3 \sqrt{3}+\pi) a^{2}+(3 \sqrt{3} \pi) l^{2}-30 a \sqrt{3 l^{2}-9 a^{2}}+6\left(12 a^{2}+l^{2}\right) \arccos \frac{\sqrt{3} a}{l}}{18 \pi a^{2}}, & \sqrt{3} \leq 2 \\ 1.0, & 2<\frac{l}{a}\end{cases}
$$

\subsubsection{Capsule dosage model}

Assume that the multi-shaped crack pattern is composed of numerous combined cells as in Figure 9, which are arranged periodically. In Figure 10, an observed parallelogram region $E_{x y}$ with the sizes of region of $x$ and $y$ is selected as the representative sampling region where $n^{2}$

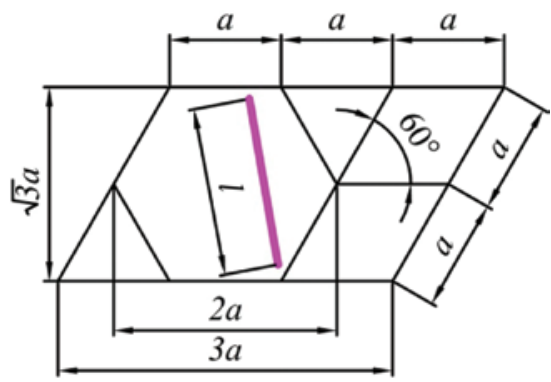

Figure 9: The combined cell in the multi-shaped crack pattern.

\subsubsection{Verification of crack-hitting probability models}

We generate a combined cell, as shown in Figure 9, and the ratios of the capsule length to the crack size $(l / a)$ are selected to be equal to $0.50,0.87,1.10$ and 1.80 , which is located in the specified ranges as listed in Table 2 . The process of verification is the same as that in Section 3.1.4. The verification results of crack-hitting probabilities by the capsules for the specified complex crack pattern are given in Figure 11. When the total number of capsules increases to about 2000 , the simulation results for crack-hitting probability models show good consistency with theoretical solutions.

\subsubsection{Verification of capsule dosage models}

Generate a region $E_{x y}$ as shown in Figure 10. Other parameters are the same as those in Section 3.1.5, and the 


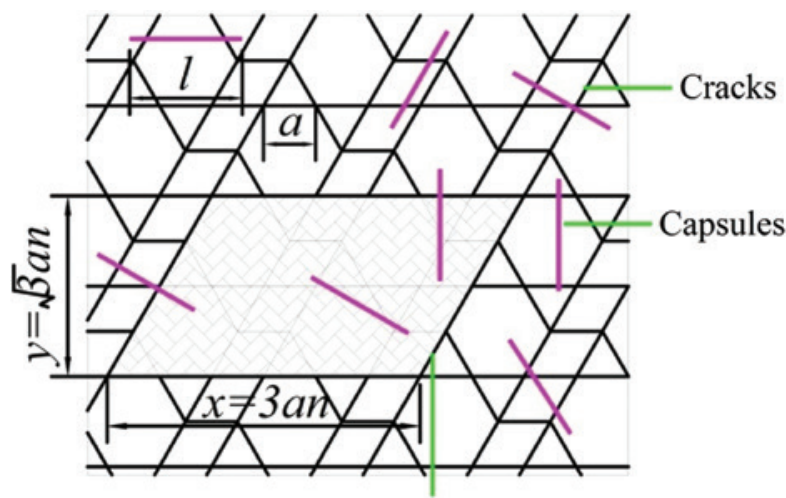

Figure 10: A parallelogram representative region $E_{x y}$ with the sizes of $x$ and $y(x=3 a n, y=\sqrt{3} a n)$.

required dosage of capsules $\left(N_{A}\right)$ is determined by Eq. (24). From the variation tendency of curves in Figure 12, it is noted that the simulation results at different ratios of the capsule length to the crack size agree with the analytical solutions from the proposed models, which verifies the reliability and rationality of the method in this study.

Figure 13 show the effect of simplified 2D crack patterns on the required number of capsules per unit area $\left(N_{A}\right)$. At the same $l / a$, the values of $N_{A}$ show significant difference for various mono-shaped crack patterns (i.e. triangular, rhombic, and hexagonal crack patterns), which illustrate the importance of the crack distribution. Meanwhile, it is found that the value of $N_{A}$ for multi-shaped crack pattern is less than those for triangular and rhombic crack patterns

Table 2: Selection of the ratio $/ / a$ for the triple-shaped crack pattern.

\begin{tabular}{lccccc}
\hline Crack pattern & $\frac{l}{a} \leq \frac{\sqrt{3}}{2}$ & $\frac{\sqrt{3}}{2}<\frac{l}{a} \leq 1$ & $1<\frac{l}{a} \leq \sqrt{3}$ & $\sqrt{3}<\frac{l}{a} \leq 2$ & $2<\frac{l}{a}$ \\
\hline $\begin{array}{l}\text { Triple-shaped } \\
\text { crack pattern }\end{array}$ & 0.50 & 0.87 & 1.10 & 1.80 & - \\
\hline
\end{tabular}

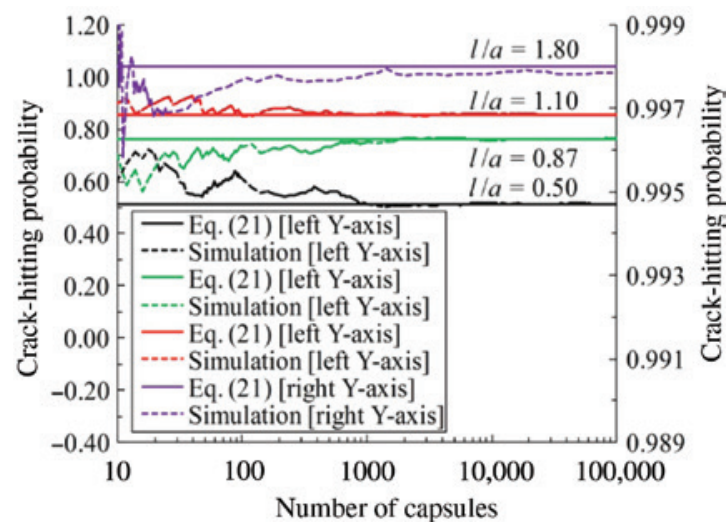

Figure 11: Verification of crack-hitting probability models for the multi-shaped crack pattern. but is higher than that for the hexagonal crack pattern. In Figure 13, the results are also compared with the analytical solution for 2D parallel cracks and orthogonal cracks in the literature [21, 22]. For the parallel crack system, the symbol $a$ here represents the distance between two neighboring parallel cracks. For the orthogonal crack system, the sides of the rectangles are equal and both are denoted by the symbol $a$. It can be seen that the value of the required number of capsules per unit area $\left(N_{A}\right)$ for multi-shaped crack pattern is larger than the values of $N_{A}$ for both parallel and orthogonal cracks. From Figure 1, the real crack distribution on the 2D plane can be characterized by various irregular convex polygons. In view of the fact that the multi-shaped crack system in this study is more approximate to the models of convex polygons compared with mono-shaped crack patterns, the corresponding $N_{A}$ would have better precision and promising application value than before. Actually, it is not hard to find that in Figure 1 the sizes of linear cracks on the surface of materials are not exactly identical. Thus, to improve the reliability of the simulated results, further

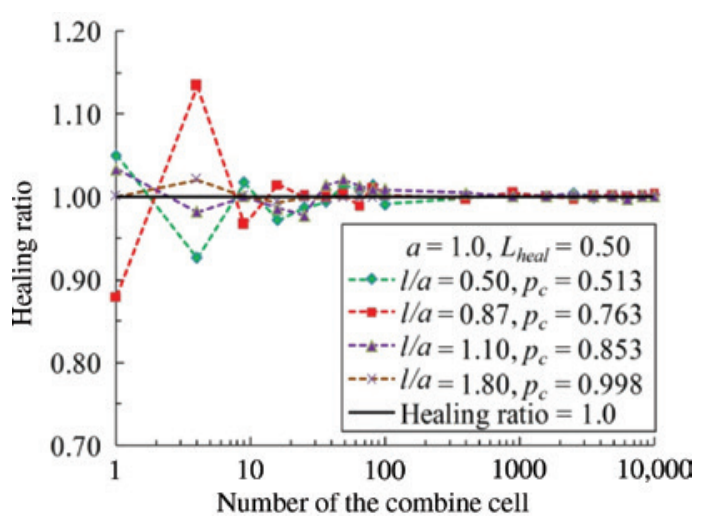

Figure 12: Verification of capsule dosage models for the multishaped crack pattern.

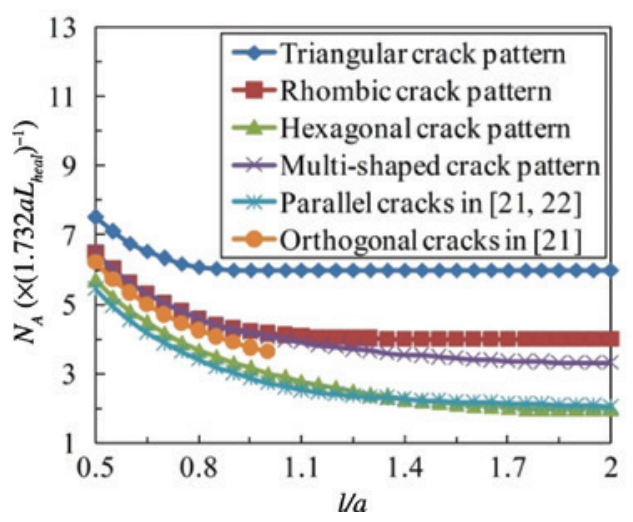

Figure 13: The minimum required number of capsules per unit area $N_{A}$ for different 2D crack patterns. 
studies on the models of multi-sized and multi-shaped convex polygons are still strongly needed.

In this article, we mainly considered 2D cracks on the plane. In fact, the majority of cracks in materials are 3D distribution. In Ref. [19], the cracks in the cement paste are simplified to a 3D plane to investigate the crack-hitting probability by the spherical capsules. Lv et al. [21] studied the crack-hitting probability by the linear capsules in materials with 3D orthogonal parallel planar cracks and zonal cracks. Strictly speaking, the above crack distributions are still simple. In recent years, 3D Voronoi tessellations [43] are widely used to model the random structure of porous media. The structures generated by using Voronoi tessellations are very similar to the 3D crack distribution in materials. If possible, these structures could also be utilized to study the crack-hitting probability by the capsules. According to Ref. [44], there is a close relationship between the crack-hitting probability and the chord length distribution function. By using this principle, current models can be easily extended to calculate the capsule dosage for complex crack systems such as Voronoi tessellations. Besides, it is worth noting that in the 3D space, various types of capsules such as cylinders [45], spheres [46-48], and spherocylinders [49] were also widely used in materials. If the influence of all of the crack patterns, crack size distribution, and capsule shapes can be included in the models, the research will be more valuable.

\section{Conclusion}

A 2D multi-shaped geometrical structure composed of triangles, rhombuses, and hexagons with specified area fractions as well as linear segments was employed to determine the minimum dosage of capsules in selfhealing materials with complex crack systems. Based on geometric measure theory, the analytical models of crackhitting probabilities and capsule dosage for the multishaped crack system were derived at various ratios of the capsule length to the crack size. The results revealed that for a given multi-shaped crack pattern, the area fraction of mono-shaped cells in the system and the ratio of the capsule length to the crack size are two key parameters determining the crack-hitting probability by the capsules and the dosage of capsules. Since the capsule length is normally fixed during the preparation stage of materials, the self-healing efficiency of materials will depend on the spatial distribution and size of the cracks. The methodology presented here can be extended to other 2D/3D complex crack networks with any arbitrary shape of capsules by using the chord length distribution function, and the solutions on capsule dosage can be derived then.

Acknowledgments: Jianjun Lin and Huisu Chen gratefully acknowledge financial support from the National Natural Science Foundation of China via Grant No. 51461135001, the Ministry of Science and Technology of China "973 plan" via Grant No. 2015CB655102, and the Fundamental Research Funds for the Central Universities via Grant No. 2242016K41054. Zhong Lv is grateful for the financial support from the National Natural Science Foundation of China via Grant No. 51408002.

\section{Appendix}

\section{The kinematic measure of a linear capsule in convex domains [40]}

Here, formulas (A.1), (A.2), and (A.3) represent the kinematic measure of a linear capsule in regular triangular, rhombic, and hexagonal cells, respectively.

For the triangular cell

$m(l)= \begin{cases}\frac{\sqrt{3} \pi a^{2}}{4}-3 a l+\frac{\sqrt{3} \pi l^{2}}{6}+\frac{3 l^{2}}{4}, & \frac{l}{a} \leq \frac{\sqrt{3}}{2} \\ \frac{\sqrt{3} \pi a^{2}}{4}-3 a l-\frac{9 a}{2} \sqrt{l^{2}-\frac{3 a^{2}}{4}}+\frac{\sqrt{3} \pi l^{2}}{6}+\frac{3 l^{2}}{4}-\left(\sqrt{3} l^{2}+\frac{3 \sqrt{3} a^{2}}{2}\right) \arccos \frac{\sqrt{3} a}{2 l}, & \frac{\sqrt{3}}{2}<\frac{l}{a} \leq 1\end{cases}$

For the rhombic cell

$$
m(l)= \begin{cases}\frac{\sqrt{3} \pi a^{2}}{2}-4 a l+l^{2}+\frac{\sqrt{3} \pi l^{2}}{18}, & \frac{l}{a} \leq \frac{\sqrt{3}}{2} \\ \frac{\sqrt{3} \pi a^{2}}{2}-4 a l+l^{2}+\frac{\sqrt{3} \pi l^{2}}{18}+5 a \sqrt{l^{2}-\frac{3 a^{2}}{4}}-\left(\frac{2 \sqrt{3} l^{2}}{3}+2 \sqrt{3} a^{2}\right) \arcsin \frac{\sqrt{3} a}{2 l}, & \frac{\sqrt{3}}{2}<\frac{l}{a} \leq 1 \\ \frac{\sqrt{3} \pi a^{2}}{3}-\frac{3 a^{2}}{4}-\frac{l^{2}}{2}-\frac{\sqrt{3} \pi l^{2}}{9}+\frac{3 a}{2} \sqrt{l^{2}-\frac{3 a^{2}}{4}}-\left(\sqrt{3} a^{2}+\frac{\sqrt{3} l^{2}}{3}\right) \arccos \frac{\sqrt{3} a}{2 l}, & 1<\frac{l}{a} \leq \sqrt{3}\end{cases}
$$


For the hexagonal cell

$$
m(l)= \begin{cases}\frac{3 \sqrt{3} \pi a^{2}}{2}-6 a l-\frac{\sqrt{3} \pi l^{2}}{6}+\frac{3 l^{2}}{2}, & \frac{l}{a} \leq 1 \\ \frac{5 \sqrt{3} \pi a^{2}}{2}+\frac{\sqrt{3} \pi l^{2}}{2}-\left(3 \sqrt{3} a^{2}+2 \sqrt{3} l^{2}\right) \arcsin \frac{\sqrt{3} a}{2 l}-\frac{9 a \sqrt{4 l^{2}-3 a^{2}}}{2}, & 1<\frac{l}{a} \leq \sqrt{3} \\ 2 \sqrt{3} \pi a^{2}+\frac{\sqrt{3} \pi l^{2}}{6}-9 a^{2}-\frac{3 l^{2}}{2}+15 a \sqrt{l^{2}-3 a^{2}}-\left(12 \sqrt{3} a^{2}+\sqrt{3} l^{2}\right) \arccos \frac{\sqrt{3} a}{l}, & \sqrt{3}<\frac{l}{a} \leq 2\end{cases}
$$

where $l$ is the length of the linear capsule and $a$ is the side length of the convex domain.

\section{References}

[1] Jaffer SJ, Hansson CM. Corros. Sci. 2008, 50, 3343-3355.

[2] Lee SY, Choo H, Liaw PK, An K, Hubbard CR. Acta Mater. 2011, 59, 495-502.

[3] Wang HL, Dai JG, Sun XY, Zhang XL. Constr. Build. Mater. 2016, 107, 216-225.

[4] Dementsov A, Privman V. Phys. A 2017, 385, 543-550.

[5] Zhu DY, Rong MZ, Zhang MQ. Prog. Polym. Sci. 2015, 49-50, 175-220.

[6] Huang HL, Ye G, Qian CX, Schlangen E. Mater. Des. 2016, 92, 499-511.

[7] Diesendruck CE, Sottos NR, Moore JS, White SR. Angew. Chem. Int. Ed. 2015, 54, 2-22.

[8] Luo M, Qian CX, Li RY. Constr. Build. Mater. 2015, 87, 1-7.

[9] van Tittelboom K, De Belie N, De Muynck W, Verstraete W. Cem. Concr. Res. 2010, 40, 157-166.

[10] Chuo TW, Liu YL. Compos. Sci. Technol. 2015, 118, 236-243.

[11] Huang L, Yi NB, Wu YP, Zhang Y, Zhang Q, Huang Y, Ma YF, Chen YS. Adv. Mater. 2013, 25, 2224-2228.

[12] White SR, Sottos NR, Geubelle PH, Moore JS, Kessler MR, Sriram SR, Brown EN, Viswanathan S. Nature 2001, 409, 794-797.

[13] Li WT, Jiang ZW, Yang ZH, Zhao N, Yuan WZ. PLoS One 2013, 8, e81616.

[14] Dong BQ, Wang YH, Fang GH, Han NX, Xing F, Lu YY. Cem. Concr. Compos. 2015, 56, 46-50.

[15] Tao Y, Chang Y, Tao YX, Yang ZG, Wu HP. Mater. Chem. Phys. 2014, 148, 778-782.

[16] Moll JL, Jin HH, Mangum CL, While SR, Scottos NR. Compos. Sci. Technol. 2013, 79, 15-20.

[17] Rule JD, Sottos NR, White SR. Polymer 2007, 48, 3520-3529.

[18] Huang HL, Ye G, Shui ZH. Constr. Build. Mater. 2014, 63, 108-118.

[19] Huang HL, Ye G. Adv. Mater. Sci. Eng. 2016, 2016, 1-10.

[20] Yuan HF, Chen HS. Comput. Concr. 2013, 11, 223-236.

[21] Lv Z, Chen HS, Yuan HF. J. Intell. Mater. Syst. Struct. 2014, 25, 47-57.

[22] Lv Z, Chen HS, Yuan HF. Sci. Eng. Compos. Mater. 2011, 18, 13-19.

[23] Lv Z, Chen HS. Smart. Mater. Struct. 2014, 23, 1-13.
[24] Monteiro PJ. Proc. Natl. Acad. Sci. USA 2006, 103, 11467-11472.

[25] Bisschop J, Wittel FK. Cem. Concr. Compos. 2011, 33, 466-473.

[26] Rajabipour F, Giannini E, Dunant C, Ideker JH, Thomas MD. Cem. Concr. Res. 2015, 76, 130-146.

[27] Liu L, Shen DJ, Chen HS, Qian ZW, Zhao HT, Jiang JH. Cem. Concr. Compos. 2014, 53, 1-9.

[28] Nagel W, Mecke J, Ohser J, Weiss V. Image Anal. Stereol. 2008, 27, 73-78.

[29] Zhao YC, Qiang LH, Yan JT. Appl. Surf. Sci. 2011, 257, 43774383.

[30] Wu ZH, Hu S, Zhou FJ. Expert Syst. Appl. 2014, 41, 1021-1030.

[31] Ohnishi T, Okada O, Shirakata H. Phys. A 2013, 392, 4127-4133.

[32] Huang HL, Ye G. In Proceedings of International RILEM Conference on Advances in Construction Materials Through Science and Engineering, Hong Kong, 2011, pp. 530-536.

[33] Solomon H. Geometric Probability, Society for Industrial and Applied Mathematics: PA, USA, 1978.

[34] Mendenhall W, Beaver RJ, Beaver BM. Introduction to Probability and Statistics, Duxbury: Belmont, 2006.

[35] Ren DL. Topics in Integral Geometry, World Scientific: Singapore, 1994.

[36] Okabe A, Boots B, Sugihara K, Chiu SN. Spatial TessellationsConcepts and Applications of Voronoi Diagrams, Wiley: New York, 1992.

[37] Lautensack C, Zuyev S. Adv. Appl. Probab. 2008, 40, 630-650.

[38] Nagel W, Weiss V. Adv. Appl. Probab. 2005, 37, 859-883.

[39] Nagel W, Weiss V. Image Anal. Stereol. 2008, 27, 29-37.

[40] Li RZ, Zhang GY. J. Wuhan. Iron. Steel. Univ. (in Chin.) 1984, 1, 106-128.

[41] Underwood EE. Quantitative Stereology, Addison-Wesley: London, 1970.

[42] Xie FF, Li DY. Acta. Math. Sci. 2011, 31B, 303-308.

[43] Xiao F, Yin XL. Comput. Math. Appl. 2016, 72, 328-348.

[44] Stoyan D, Kendall WS, Mecke J. Stochastic Geometry and Its Applications, JohnWiley \& Sons: Chichester, 1995.

[45] Lv Z, Chen HS. Comput. Mater. Sci. 2013, 68, 81-89.

[46] Perez G, Erkizia E, Gaitero JJ, Kaltzakorta I, Jiménez I, Guerrero A. Mater. Chem. Phys. 2015, 165, 39-48.

[47] Lv Z, Chen HS. Sci. Eng. Compos. Mater. 2012, 19, 1-7.

[48] Chung K, Lee S, Park M, Yoo P, Hong Y. J. Ind. Eng. Chem. 2015, 29, 330-337.

[49] Lv Z, Li SP, Chen HS. PLoS One 2017, 12, e0187299. 